\title{
Ricerche
}

\section{DALLA PAGINA ALLO SCHERMO. LE TRASFORMAZIONI DEL LIBRO E DELLA LETTURA}

\author{
di Lodovica Braida
}

Da diversi anni libri e giornali dedicano grande spazio al futuro del libro e alla rivoluzione in corso che sta portando al trasferimento del patrimonio scritto dal supporto cartaceo a quello elettronico. A differenza dell'invenzione di Gutenberg che, per quanto fosse innovativa, aveva creato pur sempre un codex, cioè un quaderno fatto di carte, la rivoluzione cui stiamo assistendo prevede la trasformazione dei supporti e delle forme attraverso cui un testo è trasmesso. Per questo, per capire le mutazioni del presente è necessario non perdere il raccordo con il passato. Solo così si possono cogliere le analogie e le differenze, le continuità e le innovazioni che hanno segnato la trasmissione della cultura scritta nel corso dei secoli, dal manoscritto al libro a stampa fino al libro digitale. Si tratta di passaggi tecnologici ma anche sociali, economici, giuridici e più ampiamente culturali.

Uno sguardo di lunga durata ci consente di riflettere sul fatto che non siamo i primi a vivere una rivoluzione nella trasmissione della cultura scritta. Altri prima di noi si trovarono di fronte a grandi cambiamenti tecnologici. Altri toccarono con mano che la tecnologia non basta, almeno nel breve periodo, a cambiare abitudini consolidate. Lo studio del passaggio dal manoscritto al libro a stampa mostra, almeno nei primi cinquant'anni di diffusione in Europa delle stamperie, più elementi di continuità che di drastiche trasformazioni. Proprio come sta accadendo oggi. Come ha scritto lo storico americano Robert Darnton, «l'esplosione delle modalità di comunicazione elettroniche è altrettanto rivoluzionaria dell'invenzione della stampa a caratteri mobili, e noi abbiamo altrettante difficoltà ad assimilarla di quante ne ebbero i lettori nel Quattrocento, quando si trovarono di fronte ai testi a stampa» ${ }^{1}$. E proprio per una disposizione umana non trascurabile, quella dell'abitudine, le nuove tecnologie non sostituiscono di colpo quelle vecchie: come il manoscritto e il libro a stampa convissero per un

1. Darnton, 2011, p. 19. Sulle grandi trasformazioni della rivoluzione digitale si veda anche Roncaglia, 2010. 
lungo periodo, così sta accadendo nella trasformazione in corso che vede la coesistenza di libri a stampa e libri digitali.

Eppure da anni passiamo da previsioni sulla morte del libro di carta a informazioni che, al contrario, sembrano andare in direzione opposta. Sembra infatti che, nonostante gli sforzi di lanciare vari tipi di e-readers, l'e-book faccia ancora fatica a entrare nelle abitudini di milioni di lettori, anche se nell'ultimo anno, in Italia, qualcosa è leggermente cambiato: il Rapporto sullo stato dell'editoria in Italia 2017 documenta come nel 2016, nel nostro Paese, il mercato e-book, nonostante sia cresciuto meno di quanto si prevedeva, sia arrivato a 81.035 "manifestazioni" (lo stesso titolo può cioè apparire in versione Pdf, ePub, ePub3, Mobipocket, ecc.), rispetto alle 62.544 del 2015. Secondo il Rapporto, il numero di titoli realmente usciti in versione e-book (escludendo quindi i dati relativi alle diverse manifestazioni di uno stesso titolo) è stato nel 2016 di 74.020, contro 62.573 titoli di libri di carta (varia adulti e ragazzi). Per la prima volta - anche se il dato assoluto è "inquinato" dalla produzione self published digitale o carta+digitale - il numero di e-book pubblicati ha superato quello dei libri di carta. Ma a fronte di questo aumento di produzione si registra un leggero calo di chi ha effettivamente letto e-book: nel 2015 erano 4,7 milioni gli italiani che dichiaravano di aver letto anche un solo e-book negli ultimi 3 mesi, mentre nel 2016 soltanto 4,2 milioni. Dunque, dopo i primi anni di forte crescita, anche la lettura di e-book presenta segnali di rallentamento ${ }^{2}$. Il $37 \%$ dei lettori acquista libri di carta ed e-book (erano il $28 \%$ nel 2015) e solo 1'1\% dichiara di leggere libri esclusivamente in formato e-book ${ }^{3}$.

Questi dati confermano che lettura del libro cartaceo e lettura su supporti diversi convivono e convivranno ancora a lungo, in Italia e, con dati diversi da Paese a Paese, in tutta Europa. E allora quale posizione assumere per comprendere ciò che sta avvenendo, senza essere in balia di previsioni fluttuanti? A questo proposito, mi sembra del tutto condivisibile la posizione dello storico francese Roger Chartier:

Tra i giudizi apocalittici che identificano [le trasformazioni in atto] come la morte dello scritto e gli apprezzamenti benevoli che colgono rassicuranti continuità, un'altra via è possibile e necessaria. Essa poggia sulla storia, non per enunciare incerte profezie, ma per comprendere meglio la coesistenza attuale (e forse destinata a durare) tra differenti modalità dello scritto - manoscritto, stampato, elettronico - e soprattutto per ricercare con più rigore come e perché nel mondo digitale sono messe in discussione le nozioni che hanno fondato la definizione dell'opera in quanto opera, il rapporto tra la scrittura, l'individualità e la proprietà intellettuale (Chartier, 2015, p. 16).

2. Lolli e Peresson, a cura di, 2017.

3. Il Rapporto sullo stato dell'editoria in Italia 2017 segnala, tra l'altro, che sempre più lo smartphone è diventato il device di riferimento per cercare e trovare informazioni, notizie, servizi e, sempre più spesso, leggere e-book e testi complessi. 
Nella riflessione che segue si farà proprio ricorso alla storia e a come la ricerca storica, e in particolare nell'ambito della storia del libro e della lettura, ci aiuti a portare l'attenzione sulla coesistenza di modalità diverse di fruire dei testi. Due mi sembrano gli ambiti di ricerca più interessanti per offrire una riflessione sul presente: gli elementi di continuità tra il manoscritto e la stampa e le trasformazioni dei modi di leggere dal passato a oggi. Partiamo dal tema della continuità.

La contrapposizione tra i due modi di produzione del libro che, a partire dal pionieristico lavoro di Marshall McLuhan (The Gutenberg Galaxy, 1962) e dal volume di Elizabeth Eisenstein (The Printing Press as an Agent of Change, 1979), aveva caratterizzato la riflessione sull'evoluzione dei media, è stata fortemente ridimensionata dagli studi degli ultimi trent'anni. Questi hanno messo in luce non solo che alcune professioni legate al mondo del manoscritto (copisti, miniatori, rubricatori, cartolai) furono coinvolte nel processo produttivo e di distribuzione del libro a stampa, ma che quest'ultimo riprendeva dai codici manoscritti sia le caratteristiche di organizzazione del testo e delle immagini, sia le caratteristiche materiali ${ }^{4}$. Anche i caratteri rivelano la continuità con le scritture fino ad allora usate dai copisti: non a caso per i primi tipografi così come per $\mathrm{i}$ lettori, la bellezza dei caratteri risiedeva in primo luogo nella leggibilità, nel fatto cioè che sembrassero "scritti con la penna". E l'imitazione era talmente ben riuscita che nel marzo del 1455 l'umanista Enea Silvio Piccolomini, al servizio di papa Niccolò V ed egli stesso futuro pontefice (Pio II), trovandosi alla fiera di Francoforte, non si accorse di avere tra le mani la prima Bibbia a stampa, la cosiddetta Bibbia a 42 linee stampata da Gutenberg a Magonza. E in una lettera al cardinale spagnolo Juan de Carvajal, Piccolomini scriveva di aver visto alcuni fascicoli di una Bibbia «di scrittura molto chiara e corretta, senza errori da nessuna parte, che Sua Signoria avrebbe letto senza fatica e senza occhiali» (Braida, 2000, p. 15).

Soltanto riconsiderando l'antica relazione tra i due media, libro manoscritto e libro a stampa, possiamo renderci conto che non si trattò di una sostituzione di un modo di produzione con un altro, ma che i due sistemi convissero in Europa per molto tempo in modo interdipendente. A lungo il calamo e il carattere furono usati per completarsi a vicenda. I primi stampatori lasciavano le iniziali in bianco e ampi margini che attendevano che il lettore scegliesse la tipologia della decorazione, in base al denaro che era disposto a spendere per un esemplare di lusso o per uno semplicemente rubricato. Quando nel 1502 lo stampatore ve-

4. Il tema della continuità tra libro manoscritto e libro a stampa è stato alla base di alcuni fondamentali studi di Armando Petrucci, a partire da Alle origini del libro moderno. Libri da banco, libri da bisaccia, libretti da mano, in «Italia Medioevale e Umanistica», XII (1969), pp. 295-313. Questo saggio è ora raccolto in Petrucci, 2017, pp. 
neziano Aldo Manuzio si rivolse con una supplica alla Repubblica veneziana per ottenere una privativa che proteggeva i suoi investimenti, annunciava che le sue «lectere greche» avevano «ligature che pareno cum calamo», belle e leggibili come se fossero uscite dalla penna di un umanista. Il massimo per uno stampatore che aveva ben presente quanto i dotti fossero abituati ai manoscritti e quanto l'avvento della stampa non avesse per nulla scoraggiato l'uso dei libri vergati «cum calamo» ${ }^{5}$.

Anche la terminologia usata e i cataloghi delle biblioteche europee sono una prova che la convivenza resistette a lungo: per indicare i manoscritti o i libri a stampa si usavano indifferentemente termini come: liber, volumen, exemplar, codex. E tale contiguità si riscontra anche nei cataloghi di biblioteca: ad esempio, il catalogo del 1605 e del 1620 di Thomas James della Bodleian Library di Oxford mescolava ancora i due tipi di prodotti. Le caratteristiche materiali dei libri che uscivano dalle tipografie in Europa nel corso del XV secolo e del primo Cinquecento, rivelano che la transizione dal manoscritto alla stampa non comportò un processo univoco e unidirezionale. Quella che è stata descritta come una printing revolution ebbe un'evoluzione lunga e, come molte rivoluzioni, il suo processo non fu lineare.

Guardando al presente, e dunque alla rivoluzione elettronica, David McKitterick ha osservato che ci troviamo, da un lato, di fronte a un culto del testo decostruito e dall'altro a una reazione di fronte al pericolo di una frammentazione testuale. In questa prospettiva, la stampa è percepita come "a fixative medium" (un mezzo cioè che tende a fissare il testo) e dunque con un forte ruolo di stabilità (McKitterick, 2005). In realtà se si analizzano i libri a caratteri mobili e la tipologia della loro produzione si ha, al contrario, l'impressione di una certa instabilità. Come ha mostrato il bibliografo di origine neozelandese Donald F. McKenzie, nella routine quotidiana del lavoro in tipografia d'antico regime, l'incertezza e l'instabilità nella produzione erano superiori alla certezza e alla stabilità. Prendendo in considerazione i registri della stamperia dell'Università di Cambridge, emergono dati dettagliati sulla divisione del lavoro e sul ruolo delle diverse figure sociali impegnate alla composizione e ai torchi. A tali fonti McKenzie affianca la documentazione sull'attività tipografica di William Bowyer attivo a Londra tra il 1730 e il 1739 . Ne risulta che gli uomini lavoravano a cottimo e quindi la produttività variava fortemente da un operaio all'altro. Dall'analisi dei fogli tirati emerge poi che era molto diffuso il sistema di stampare simultaneamente più libri per poter sfruttare al meglio i torchi e la manodopera. Dunque le norme per stabilire l'ideal copy text - al centro delle preoc-

5. Per la bibliografia su Manuzio rimando alla voce di Mario Infelise, Manuzio, Aldo, il Vecchio, in Dizionario Biografico degli Italiani, vol. 69, 2007, pp. 236-245. Mi limito qui a citare soltanto alcuni contributi essenziali: per un'analisi complessiva dell'attività di Aldo, Lowry, 1984; Dionisotti, 1995. 
cupazioni della bibliografia analitica anglosassone -, al fine di individuare il testo il più vicino possibile alla volontà dell'autore, entrano in crisi nel momento in cui emergono "le variabili collaterali" di un modo di produzione nel quale l'unica norma era, paradossalmente, "la normalità della non uniformità"6.

Negli studi sul teatro inglese in età moderna e in particolare sulle opere di Congreve stampate da Jacob Tonson nel 1710 in presenza dell'autore, lo stesso McKenzie mostra come le trasformazioni formali e materiali (ad esempio nella scelta di un formato più piccolo, dall'in-quarto all'in-ottavo, la numerazione delle scene, l'indicazione a margine del personaggio che sta parlando) abbiano avuto conseguenze importanti sullo statuto dell'opera. Il testo restava lo stesso, ma l'edizione di Tonson rendeva possibile una fruizione diversa da quelle precedenti, consentendo una lettura più agevole, senza perdere nulla della tipologia dell'opera ${ }^{7}$.

La possibilità della variazione del testo era sempre dietro l'angolo in ogni fase della produzione: gli stessi errata corrige sono una testimonianza della variante in tipografia, e non solo nel primo secolo di stampa, ma per tutto il tempo dell'antico regime tipografico. Solo per fare un esempio, nel 1702 Pierre Bayle aggiungeva una lista di errata nel primo volume del suo Dictionnaire historique et critique, avvertendo i lettori: «Notez que quelques-unes des fautes marquées dans cet Errata \& ci-dessous ne se trouvent pas dans tous les exemplaires».

Dunque la tanto enfatizzata "standardizzazione" che secondo Elizabeth Eisenstein avrebbe caratterizzato la trasmissione dei testi a stampa, in realtà deve essere ridimensionata, dal momento che non sempre i contemporanei percepivano quella polarizzazione tra un prodotto non standardizzato (il manoscritto) e un prodotto standardizzato (il libro a stampa). A colpire gli osservatori del XV secolo era piuttosto il processo di accelerazione della produzione consentito dalla stampa e il potere di conservazione del sapere che la stampa portava con sé: l'umanista Guillaume Fichet, professore alla Sorbona che aveva fatto arrivare a Parigi i primi due tipografi tedeschi (1470), osservava: «Sono stati divinizzati Bacco e Cerere per avere insegnato all'umanità l'uso del vino e del pane; ma l'invenzione di Gutenberg è ancora più grande e divina, perché egli ha inciso dei caratteri con i quali tutto ciò che si pensa e si dice può essere impresso $\mathrm{e}$ conservato alla memoria dei posteri» (Braida, 2000, p. 4).

Man mano che il libro a stampa assumeva una sua identità, i lettori sempre più furono colpiti anche dalla leggibilità dei testi. Quando Aldo Manuzio, all'inizio del Cinquecento, per la prima volta fece uscire i classici latini e greci in un formato tascabile, i suoi lettori gli furono grati. Ed erano i lettori colti di tutta Europa. Fino ad allora nessuno aveva ancora pubblicato gli autori greci

6. McKenzie,1966; Id., 2003, p. 35.

7. Id., 2004. 
che sono alla base della cultura umanistica. E proprio le osservazioni dei lettori dei libri di Manuzio ci danno un'idea che ad attrarli era il fatto che fossero maneggevoli, che si potessero finalmente leggere facilmente, senza il peso e la scomodità delle edizioni in formato grande. «Tu ti sei dato la pena - gli scriveva il duca d'Atri - di mettere [gli autori greci e latini] alla nostra portata; ora possiamo conoscerli e frequentarli nell'intimità». E ancora: «I tuoi libri - così maneggevoli da poterli usare camminando - sono diventati per me un piacere speciale», gli scriveva nel 1501, da Budapest, Sigismondo Thurzó, segretario del re di Ungheria e suo cliente e amico ${ }^{8}$. Che i libri fossero estremamente comodi e leggeri lo rivelano anche alcuni dipinti, come il bellissimo giovane con il libro verde di Giorgione. L'uomo è ritratto in piedi, mentre tiene in mano, senza sforzo, un libro aperto, inequivocabilmente un'edizione aldina in ottavo. Con tutta probabilità il giovane legge mentre sta camminando. Ha appena alzato lo sguardo dalla pagina ed è assorto nella sua lettura solitaria 9 .

Va detto che in molti casi i testi a stampa erano ben lungi dall'essere completi o perfetti: in molti casi troviamo libri con parti di testo non stampate o stampate in un ordine sbagliato. E nei casi in cui un libro fosse rimasto invenduto o che il suo acquirente non lo avesse fatto decorare o miniare, gli spazi previsti per l'intervento della mano dell'uomo rimanevano bianchi. E oggi a noi sembrano incompiuti, ma per i cartolai del tempo era del tutto normale. Anche quando la tecnica cambiò, imperfezioni, varianti, incoerenze, erano fortemente connaturate al libro e al giornale a stampa. Verso la fine del Settecento, gli stampatori francesi Pierre e Firmin Didot investirono nei nuovi macchinari per la stereotipia (e dunque stampavano libri non direttamente da caratteri, ma da lastre). Nella presentazione dei loro testi classici, gli editori sottolineavano «l'extrême importance de la correction dans les textes» che la nuova tecnica consentiva. Dunque facevano riferimento a un mondo della stampa in cui era possibile una certa flessibilità, potendosi rifare, in modo rapido e «straordinariamente economico» (McKitterick, 2005, p. 241), intere lastre, qualora si fossero riscontrati degli errori. Ancora una volta, una tecnica che a noi sembra piuttosto fissa (dal momento che non si possono apportare delle correzioni una volta fusa la forma), era qui descritta con entusiasmo per la sua mobilità.

Questi esempi ci inducono a riflettere su quanto possa cambiare la percezione del testo e del supporto su cui è trasmesso, a seconda dei diversi momenti storici, dei condizionamenti e delle trasformazioni sociali in corso. Il nostro modo di giudicare il passato dipende dal nostro modo di guardare al presente,

8. Braida, 2000, p. 86. La lettera a Manuzio di Thurzó è pubblicata in de Nolhac, 1887, pp. 247-299.

9. Per la riproduzione di questo quadro cfr. il catalogo della mostra Pietro Bembo $e$ l'invenzione del Rinascimento, a cura di G. Beltramini et al., 2013, p. 135 (si veda anche la scheda di A. Ballarin, ibidem, pp. 147-150). 
dalle nostre certezze e dalle nostre paure. Allora per esempio, percependo le parole sullo schermo del nostro computer o del nostro tablet come flessibili e mobili, siamo tentati di giudicare il mondo del libro a stampa come fisso e immutabile, quando invece per secoli, come ha sottolineato McKitterick, è stato infinitamente mobile e giudicato come tale (ibidem, p. 222).

Il secondo ambito di ricerca che ci aiuta a capire meglio le trasformazioni cui stiamo assistendo è quello della lettura. Proprio i recenti studi sulla storia della lettura hanno mostrato che le innovazioni tecnologiche non hanno cambiato, in poco tempo, abitudini fortemente consolidate. Tutte le trasformazioni legate all'uso dei libri furono trasformazioni lente, in cui le acquisizioni moderne gradualmente si affiancarono a quelle antiche.

L'«appropriazione» che ogni lettore fa del testo e il ruolo della materialità attraverso cui i testi sono trasmessi sono stati temi centrali negli studi che hanno rinnovato la storia del libro, fino agli anni Ottanta poco attenta al problema della lettura. A sottolineare l'importanza di studiare la lettura, e non solo la produzione del libro, fu uno studioso francese, Michel de Certeau, che nel 1980 scriveva che la lettura doveva essere considerata come «un'altra produzione», «silenziosa e quasi invisibile» (de Certeau, 1990, pp. 35-36): quella appunto del singolo lettore o della comunità di lettori che si avvicinano al testo secondo le proprie capacità. Da allora non sono mancate ricerche non solo sulle occasioni, sui luoghi e sulle circostanze in cui la lettura avviene, ma anche sull'universo culturale di singoli lettori e sui dispositivi testuali e materiali che possono condizionare la lettura.

La svolta metodologica è stata rappresentata da un libro prezioso, fondativo della ricerca sulla lettura, come L'apparition du livre di Lucien Febvre e di Henri-Jean Martin lo era stato, nel 1958, per la storia sociale del libro. Si tratta della Storia della lettura nel mondo occidentale, diretta da Guglielmo Cavallo e da Roger Chartier (1995). Questo libro ha avuto meriti grandissimi: in primo luogo le ricerche sulla lettura in Europa dall'antichità a oggi ci hanno insegnato definitivamente che la lettura non è un atto astratto ma che si concretizza in pratiche e in luoghi in cui l'atto di leggere avviene. La lettura non è cioè un gesto che ha attraversato i secoli senza cambiare, ma è una pratica che ha una sua storia, con le sue continuità e le sue fratture. In essa si individuano svolte che non coincidono soltanto con quelle che hanno caratterizzato le forme assunte dalla cultura scritta e le tecniche della sua riproduzione.

Oltre all'invenzione e alla diffusione della stampa, anche altri momenti cruciali trasformarono la fruizione dei testi. Ad esempio, noi leggiamo in modo silenzioso, ma non è sempre stato così. Oggi la lettura silenziosa è una pratica per noi talmente interiorizzata che neppure ci rendiamo più conto che è stata una conquista relativamente recente, una conquista che si è affermata lentamente nell'Europa occidentale. Dall'antichità fino all'età moderna, quando si parlava di lettura si dava per scontato che essa avvenisse ad alta voce. Come si è arrivati 
alla lettura solitaria e silenziosa, fatta con i soli occhi? Si è arrivati per tappe successive, dapprima timidamente $\mathrm{e}$ in ambienti ristretti (le comunità monastiche) e, a partire dal Settecento, con una sorta di accelerazione che ha consentito che non si tornasse più indietro: «Allora in Occidente si superò quello spartiacque al di là del quale, in una parte consistente della popolazione, la lettura divenne quale essa è oggi, cioè veloce e silenziosa, interamente demandata alla vista» ${ }^{10}$. Pensiamo a come venivano letti i romanzi. Probabilmente, secondo Rosamaria Loretelli, senza la diffusione della lettura silenziosa il romanzo non avrebbe avuto il radicamento e il successo che ha conosciuto dal Settecento in poi in Europa. Fu in Inghilterra che si verificarono le condizioni, prima che altrove, perché il romanzo trionfasse. La definitiva standardizzazione del libro, la protezione del ruolo autoriale con la legge sul copyright (1709) e l'attenzione per l'uniformità grafica e linguistica resero la lettura sempre più agevole. E che i romanzi potessero trasformarsi in piacevoli trappole che catturavano i loro lettori e le loro lettrici fino alla fine della storia se ne accorsero gli editori per i quali i romanzi costituirono un grande affare. Ma quale era il segreto per affascinare migliaia di lettori e lettrici, fino a suscitare una vera e propria passione di leggere? Una possibile risposta la troviamo nei Philosophical Essays Concerning Human Understanding (1748) del filosofo scozzese David Hume. A suo avviso, gli autori possono, attraverso adeguate tecniche narrative, suscitare in chi legge symphaty, ovvero empatia. Occorre che il lettore sia messo nelle condizioni di immaginare il più possibile i luoghi e le circostanze in cui si muovono i personaggi, e non solo le azioni che compiono. I fatti devono essere mostrati nei dettagli e non come se li vedessimo da una carrozza in corsa, come accade nell'Henriade di Voltaire «dove gli eventi - scrive Hume - scorrono con tale rapidità, che a malapena abbiamo l'agio di essere messi a conoscenza di una scena o di un'azione» ${ }^{11}$.

Ed è esattamente il tipo di narrazione che i grandi romanzieri inglesi, e successivamente quelli tedeschi, francesi e italiani, introducono con forza, una narrazione che finiva per facilitare una lettura veloce e silenziosa e, consentendo di concentrarsi subito sui contenuti, «costruiva una relazione con l'interiorità e faceva sentire al lettore il rapporto con l'opera e il suo autore come qualcosa di assolutamente privato e intimo» ${ }^{12}$. Così si spiega perché, nelle Affinità elettive (I ed. 1809) di Goethe, Ottilia, nonostante ascoltasse la lettura ad alta voce di Edoardo, preferisse però scorrere le pagine del libro da sola: «si fidava più dei propri occhi che delle labbra altrui» (Goethe, 1999, p. 64).

Ma per le donne a lungo contò, più che la lettura solitaria, la lettura condivi$\mathrm{sa}$, ad alta voce nelle serate conviviali o nei salotti, come ha mostrato un impor-

10. Loretelli, 2010, p. 5.

11. La citazione è tratta da ibidem, p. 137.

12. Ibidem, p. 62. 
tante volume curato da Maria Luisa Betri ed Elena Brambilla. Proprio le ricerche coordinate dalle due storiche sottolineano «il ruolo assunto [...], tra fine Seicento ed inizio Novecento, da figure femminili mediatrici e organizzatrici di cultura nei salotti aristocratici e poi borghesi, in una sfera distinta sia da quella dell'intimità domestica, sia da quella propriamente politica». Grazie alle animatrici di raffinati circoli intellettuali di impronta aristocratica si crearono spazi in cui si discuteva di libri, di giornali, si prendeva posizione su temi filosofici, letterari e politici e si creavano le condizioni per rendere sempre più «accettabile la figura di donna colta, educata e letterata, in grado di influenzare mentalità e atteggiamenti collettivi e di imporre una più equilibrata concezione dei rapporti di genere» ${ }^{13}$.

Ma le donne e gli uomini che condividevano queste esperienze culturali leggevano pur sempre sullo stesso supporto: il libro o il giornale di carta, e più tardi il feuilleton o il fotoromanzo che si procuravano nel modo tradizionale, attraverso l'acquisto in libreria o dal giornalaio, o in alcune città attraverso le copie che potevano affittare nei gabinetti di lettura o prendere in prestito in biblioteca. Oggi la rivoluzione cui stiamo assistendo, che riguarda la trasmissione elettronica dei testi, è ben più radicale perché, per la prima volta nella storia della cultura scritta, ci troviamo di fronte a un cambiamento che avviene simultaneamente su più piani e che prevede la trasformazione dei supporti della scrittura, della tecnica della sua riproduzione e della sua diffusione, e ovviamente della modalità di lettura.

Il passaggio dal supporto cartaceo a quello elettronico ci induce a riflettere sul fatto che leggere lo stesso testo su un supporto diverso non è la stessa cosa. Come i numerosi studi di bibliografia analitica hanno mostrato, le forme diverse attraverso cui un testo è trasmesso, a partire dalle sue caratteristiche materiali, ma anche dalla scelta di inserire o meno certi elementi paratestuali, possono condizionare in modo decisivo la lettura e dunque la ricezione ${ }^{14}$.

Il concetto di paratestualità si ridefinisce in relazione alla forma e alla tecnologia attraverso cui il testo è trasmesso, e nel caso del testo digitale tale definizione diventa piuttosto ambigua, anche se, per analogia con il mondo del libro a stampa, possiamo definire paratesti le mappe dei siti e i link per la navigazione. Va detto poi che mentre nel libro a stampa è relativamente facile distinguere il testo dal paratesto, non è così per l'ipertesto, dove ogni caso è diverso dall'altro: «Alcuni ipertesti fanno apparire i link come paratestuali, come aggiunte al testo principale, mentre altri fanno sì che i link appaiano così essenziali da sembrare essenzialmente testuali» ${ }^{15}$. 
Anche queste considerazioni sul rapporto tra testo e paratesto nel web ci inducono a riflettere sul fatto che i supporti materiali che ci trasmettono i testi non sono elementi insignificanti, ma influenzano le pratiche di lettura e gli usi degli stessi testi. Esiste però nel nostro modo di concepire la cultura scritta una sorta di paradosso: da una parte le opere sono mobili, trasformate dalle traduzioni, dagli adattamenti, dalle edizioni diverse, dall'altra i testi nella tradizione letteraria vengono idealizzati, come se esistessero indipendentemente dalla materialità del supporto che li rende fruibili ai lettori di tempi e generazioni diverse (Chartier, 2006, p. VII). Le ricerche sulla lettura e la nostra esperienza ci rivelano che la lettura non è per niente un incontro astratto e disincarnato tra mente del lettore e parole immateriali, ma è piuttosto la relazione tra mente e corpo in situazioni concrete. Siamo sensibili al formato dei libri, alle loro illustrazioni, alla loro materialità e non solo ai contenuti di cui sono portatori. E se leggere un testo su un e-reader non è ovviamente la stessa cosa che leggerlo sulla carta, chi costruisce gli e-reader, proprio perché la lettura è una pratica abitudinaria, ha cercato di imitare, nella forma in cui il testo viene fruito, qualcosa che si riallaccia all'eredità del libro, riproducendo addirittura il fruscio delle pagine, $\mathrm{o}$ in certi casi il grigiore della carta riciclata, un po' come gli stampatori del XV secolo cercavano di imitare l'impaginazione e le scritture dei manoscritti.

Nessuno nega che la rivoluzione elettronica cui assistiamo apra grandi possibilità ${ }^{16}$. Milioni di libri antichi o non più in commercio sono stati digitalizzati e messi a disposizione di tutti (si pensi a Google Books), in alcuni casi raccogliendo patrimoni di un'intera cultura (si pensi a Gallica), in altri casi biblioteche o collezioni particolari. Si tratta di un'operazione fondamentale sia per la conservazione di quei testi che per la loro fruizione. Occorre però essere consapevoli che i libri dell'era gutenberghiana o usciti prima della rivoluzione digitale, disponibili ora su un supporto elettronico, diventano fruibili separatamente dalle forme, manoscritte o a stampa, che hanno contribuito a costruire i loro significati storici (Braida, 2018). È necessario dunque riflettere anche sulle conseguenze che può avere una ricerca scollegata dalla materialità del testo.

Per documentare la storicità dei testi è fondamentale che si conservino le diverse forme attraverso cui un testo è diventato libro, disco, cd o altro ancora, per un pubblico di élite o per un pubblico più ampio, per adulti o per bambini. $\mathrm{E}$ che non ci si limiti a conservare l'ultima edizione, eliminando tutte le altre, come è accaduto per i generi di larga circolazione, e in primo luogo per i libri scolastici. Il ruolo degli archivi e delle biblioteche come deposito della memoria diventa ancora più importante oggi, in una società in cui si stanno moltiplicando

16. Sul futuro del libro di carta, sulla rivoluzione in corso che sta portando al trasferimento del patrimonio scritto dal supporto cartaceo a quello elettronico, sul rischio di affidare la digitalizzazione a grandi società come Google Book Search, cfr. R. Darnton, Il futuro del libro, cit., cap. I. 
le tipologie documentarie e in cui non si tratta solo di acquisire e conservare documenti, ma di far posto a supporti materiali nuovi ${ }^{17}$.

\section{Riferimenti bibliografici}

Beltramini G., Gasparotto D. e Tura A., a cura di (2013), Pietro Bembo e l'invenzione del Rinascimento, Catalogo della mostra (Padova, 2 febbraio-19 maggio 2013), Venezia, Marsilio.

Betri M. L. e Brambilla E., a cura di (2004), Salotti e ruolo femminile in Italia tra fine Seicento e primo Novecento, Venezia, Marsilio.

Braida L. (2000), Stampa e cultura in Europa tra XV e XVI secolo, Roma-Bari, Laterza.

Ead. (2018), Archivi culturali tra memoria d'autore e memoria d'editore, in «Letteratura e Letterature», 12, 2018, pp. 55-64.

Cavallo G. e Chartier R., a cura di (1995), Storia della lettura nel mondo occidentale, Roma-Bari, Laterza.

Certeau, de M. (1990), L'invention du quotidien, 1. Arts de faire, nuova edizione critica a cura di Giard L., Parigi, Gallimard.

Chartier R. (2006), Inscrivere e cancellare. Cultura scritta e letteratura dall'XI al XVIII secolo, Laterza, Roma-Bari, (ed. or. Parigi, 2005).

Id. (2015), La mano dell'autore, la mente dello stampatore. Cultura e scrittura nell'Europa moderna, Roma, Carocci (I ed. Cambridge, 2014).

Darnton R. (2011), Il futuro del libro, Milano, Adelphi (I ed. New York, 2009).

Dionisotti C. (1995), Aldo Manuzio umanista e editore, Milano, Il Polifilo.

Eisenstein E. L. (1979), The Printing Press as an Agent of Change: Communications and Cultural Transformations in Early-Modern Europe, 2 voll., New York, Cambridge University Press.

Giuva L., Vitali S. e Zanni Rosiello I. (2007), Il potere degli archivi. Usi del passato e difesa dei diritti nella società contemporanea, Milano, Bruno Mondadori.

Goethe J. W. (1999), Le affinità elettive, introduzione e traduzione di Cusatelli G., Milano, Garzanti (I ed. 1809).

Infelise M. (2007), Manuzio, Aldo, il Vecchio, in Dizionario Biografico degli Italiani, vol. 69, Roma, Istituto dell'Enciclopedia Italiana, pp. 236-245.

Landow G. P. (2005), L'ipertesto: testo o paratesto?, in Santoro M. e Tavoni M. G., a cura di (2005), I dintorni del testo. Approcci alle periferie del libro, Atti del Convegno internazionale (Roma, 15-17 novembre 2004; Bologna, 18-19 novembre 2004), vol. I, Roma, Edizioni dell'Ateneo, pp. 17-29.

Lolli A. e Peresson G., a cura di (2017), Rapporto sullo stato dell'editoria in Italia 2017, e-book, Ediser.

Loretelli R. (2010), L'invenzione del romanzo. Dall'oralità alla lettura silenziosa, Roma-Bari, Laterza.

Lowry M. (1984), Il mondo di Aldo Manuzio. Affari e cultura nella Venezia del Rinascimento, Roma, Il Veltro (ed. or. Oxford, 1979).

17. Su questi temi cfr. Giuva, Vitali e Zanni Rosiello, 2007. 
Dalla pagina allo schermo. Le trasformazioni del libro e della lettura

McKenzie D. F. (1966), The Cambridge University Press, 1696-1712, Cambridge, Cambridge University Press.

Id. (1999), Bibliografia e sociologia dei testi, Milano, Ed. S. Bonnard, (I ed. Londra, 1986).

Id. (2003), Stampatori della mente e altri saggi, Milano, Ed. S. Bonnard (I ed. 1969), p. 35.

Id. (2004), Di Shakespeare e Congreve, Milano, Ed. S. Bonnard.

McKitterick D. (2005), Testo stampato e testo manoscritto. Un rapporto difficile, 14501830, Milano, Ed. S. Bonnard (I ed. Cambridge University Press, 2003).

McLuhan M. (1962), The Gutenberg Galaxy: The Making of Typographic Man, Toronto, University of Toronto Press.

Nolhac, de P. (1887), Les correspondants d'Alde Manuce: matériaux nouveaux d'histoire littéraire (1483-1514), in «Studi e Documenti di Storia e Diritto», a. VIII (1887), pp. 247-299.

Petrucci A. (1969), Alle origini del libro moderno. Libri da banco, libri da bisaccia, libretti da mano, in «Italia Medioevale e Umanistica», XII (1969), pp. 295-313, ora in Id. (2017), Letteratura italiana: una storia attraverso la scrittura, Roma, Carocci, pp.

Roncaglia G. (2010), La quarta rivoluzione. Sei lezioni sul futuro del libro, Roma-Bari, Laterza.

Tavoni M. G. (2009), Circumnavigare il testo: gli indici in età moderna, Napoli, Liguori. 